\title{
A novel parametric approach to mine gene regulatory relationship from microarray datasets
}

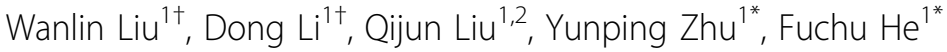 \\ From The $21^{\text {st }}$ International Conference on Genome Informatics (GIW2010) \\ Hangzhou, People's Republic of China. 16-18 December 2010
}

\begin{abstract}
Background: Microarray has been widely used to measure the gene expression level on the genome scale in the current decade. Many algorithms have been developed to reconstruct gene regulatory networks based on microarray data. Unfortunately, most of these models and algorithms focus on global properties of the expression of genes in regulatory networks. And few of them are able to offer intuitive parameters. We wonder whether some simple but basic characteristics of microarray datasets can be found to identify the potential gene regulatory relationship.

Results: Based on expression correlation, expression level variation and vectors derived from microarray expression levels, we first introduced several novel parameters to measure the characters of regulating gene pairs.

Subsequently, we used the naïve Bayesian network to integrate these features as well as the functional coannotation between transcription factors and their target genes. Then, based on the character of time-delay from the expression profile, we were able to predict the existence and direction of the regulatory relationship respectively.

Conclusions: Several novel parameters have been proposed and integrated to identify the regulatory relationship. This new model is proved to be of higher efficacy than that of individual features. It is believed that our parametric approach can serve as a fast approach for regulatory relationship mining.
\end{abstract}

\section{Background}

Gene regulation, a basic process of organisms, is important for systems biology research. Gene regulatory relationship mining can help identify the complicated regulatory networks, uncover the regulatory patterns in the cell, and expand the systematic view of biological processes.

In the past decade, as a novel high-throughput method, microarray has been widely used in genome wide research. Therefore, many algorithms have also been introduced in this field to construct gene regulatory networks based on microarray data.

\footnotetext{
* Correspondence: zhuyp@hupo.org.cn; hefc@nic.bmi.ac.cn

† Contributed equally

'State Key Laboratory of Proteomics, Beijing Proteome Research Center,

Beijing Institute of Radiation Medicine, Beijing 102206, China

Full list of author information is available at the end of the article
}

A basic hypothesis among these approaches is that the variation of expression levels of transcription factors (TF) will affect expression levels of its target genes (TGs) through the regulatory relationships. In other words, the expression profiles of TF and its TGs are somewhat interrelated. Consequently, measuring the correlation of the expression profiles represented by microarrays, especially time-series microarrays, has become a natural consideration.

Some of the previous work has contributed to this task [1]. According to the characters of the models, the algorithms can be broadly classified into several different categories.

First, clustering algorithms are basic and simple methods, based on the similarity of the expression levels of TF and its TGs [2,3]. Meanwhile, some graph models are used, such as classical graphical Gaussian models [4], and the coexpression graph-based approach [5]. 
Secondly, a series of network models has been widely used, such as Boolean network [6-8], naïve Bayesian network [9], and dynamic Bayesian network [10-12]. These methods are considered to be the mainstream gene regulatory constructing methods. Besides, ODE (ordinary differential equation) [13], NDE (nonlinear differential equation) [14] and pDE (partial differential equations) have also been introduced, which can adjust the parameters of differential equations manually to the biological process. The mutual information method is gaining popularity $[15,16]$ and is used to measure the entropy of the whole system.

However, most of these complex models and algorithms focus on global properties, and some of them could not offer parameters. This leaves us wondering whether some simple but potentially probable basic characteristics could be uncovered.

As the first step, we tested the basic Pearson correlation coefficient, PCC [17] to outline the relationship between gene regulatory relationships and the expression level represented by microarray. Generally, the existence of regulatory relationship is more likely while the absolute value of the PCC is larger. There are, however, some exceptions (see Additional file 1). In order to handle this problem, several indicators or parameters should be introduced. During correlation analysis, we found it necessary to take into account the variation of expression levels. Therefore, we measured the variation characters using both expression level differences (ELD) and differences of average - standard deviation $(\Delta$ mean $-\Delta \delta)$ parameter groups. These parameters, when combined with PCC, can effectively improve the accuracy of prediction.

Moreover, we considered expression level vectors mapped on TF-TG expression level space. Given the property of time-series gene expression relationships, it is naturally assumed that different expression relationships might relate to the vectors in different quadrants. Therefore we calculated the sum vectors in different quadrants respectively and chose the representative vector as the main vector of one TF-TG pair. Afterwards, the modulus - argument $(|x|-\theta)$ parameter group was fixed.

In addition, we analyzed functional co-annotation of transcription factors and target genes before selecting the GO score as another parameter. The parameters will be integrated to perform a better prediction. Finally, we selected Bayesian models combined with joint likelihood ratio to integrate all the parameters and achieved a better performance (See Figure 1).

\section{Results}

Construction of multiple features to mine regulatory relationships

\section{Variation of the expression levels}

To deal with exceptions during the measurement by Pearson correlation coefficient, other elements besides PCC should be considered. Taking into account the levels of expression strength, we supposed that the similarity may relate to the distribution range of the variation of expression levels. e.g., the smaller the variation range of the expression levels of tested TF-TG is, the

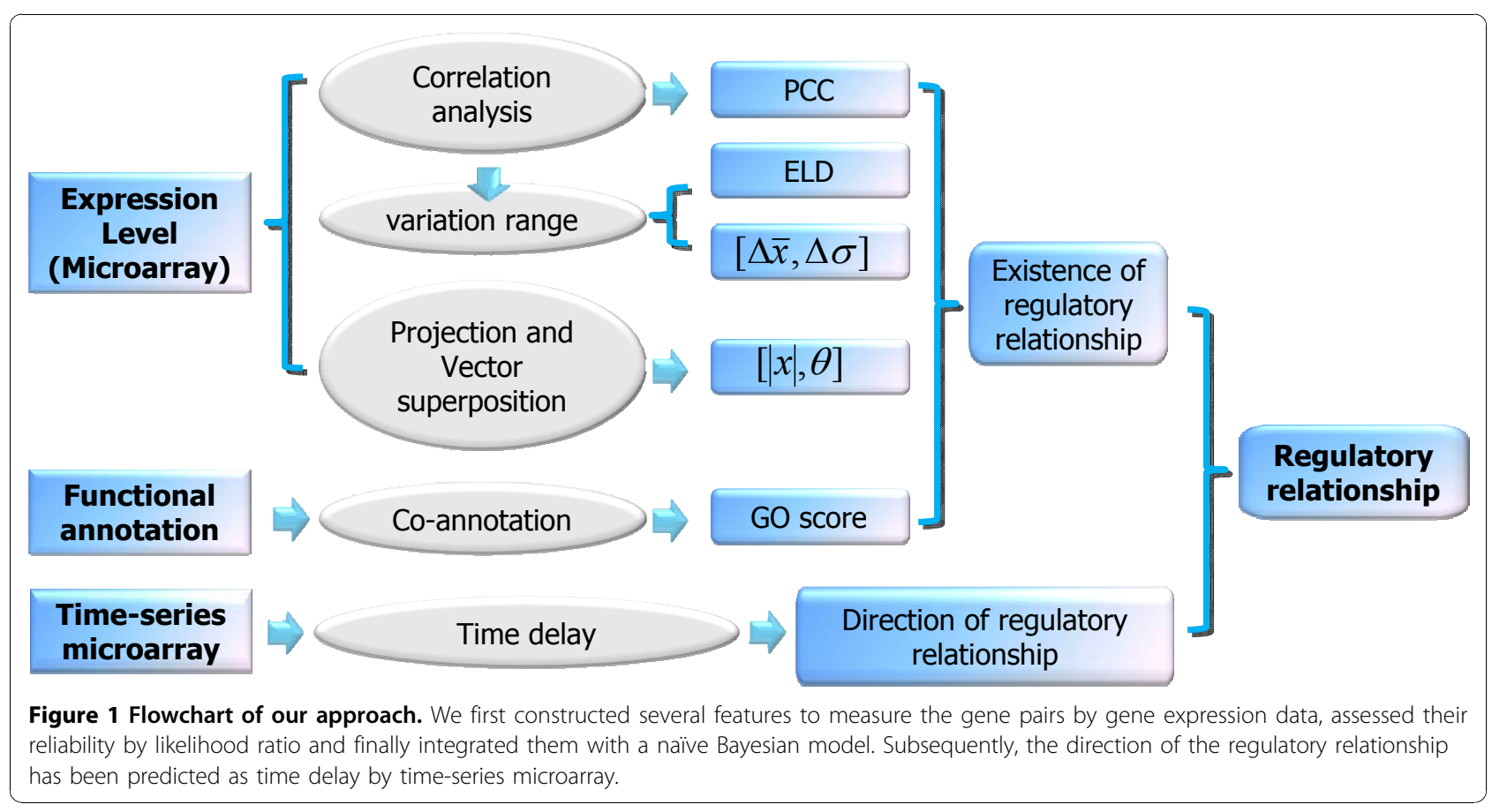


more the pair is likely to be strongly correlated (See Additional file 1).

During the analysis of the practical yeast microarray data, we recognized the necessity to take into account the dynamic range of expression levels. In order to utilize the character of time-series profiles, we measured the variation of the expression levels (series of data points). We defined a mapping of the expression levels onto the variation space (Figure 2) before we calculated the Euclidean distances between the mapped points of the original expression levels. We named the calculated Euclidean distances as the distance of expression levels' differences (ELD). This criterion can effectively reduce false negative prediction based on PCC only.

$$
E L D=\sqrt{\left(b_{x}-a_{x}\right)^{2}+\left(b_{y}-a_{y}\right)^{2}}
$$

To further study the statistic feature of expression levels, the standard deviation and their mean value were both used. The differences in the mean value and the standard deviation between TF and its TG were calculated, as in the case of another parameter group $\Delta$ mean$\Delta \delta$.

\section{Modulus and angle of expression level vector by vector analysis}

Vector analysis was used to compare gene expression responses between different experimental backgrounds [18] according to a simple principle. The change of the gene expression against the two experimental backgrounds is represented by a vector. Both up- or downregulation and the regulatory intensity can be showed. The various sectors of the Cartesian plane will correspond to various prototypical behaviours of genes.

Here we considered an extension of vector analysis, mapping expression levels under each condition or time point in the coordination system of TF-TG's expression levels. Moreover, we attempted to infer the expression patterns such as correlation or inversion with or without time-shift.

Given the property of time-series gene expression relationships, it can be naturally assumed that different expression relationships might correspond to the vectors in different quadrants. So we calculated the sum vectors in different quadrants respectively before choosing the vector which has the largest modulus as the main representative vector of one TF-TG pair (Figure 3). The representative features can be grasped and amplified by vector analysis.

\section{GO coannotation score}

It is known that the regulatory relationship emerges in the same biological process. Here we used the Gene Ontology (GO) classification system to define the measurement of functional similarity. The GO annotation term has several hierarchical grades, and coannotation terms can be organized as a tree so that all the leaves of the tree represent the coannotation strength. A leaf with deeper (more detailed) annotation denotes stronger coannotation strength and will be assigned a higher score according to the grade of the GO term. Besides, the divergence of branches of leaves has to be considered. For example, two seven-grade coannotation terms are derived from the same five-grade node, and another two seven-grade coannotation terms do not have any common parent node. Counting the scores of the pairs according to the grade of their leaves alone will obtain the same but an obviously unfair result. So the duplicated count produced by the same parent term should be excluded. That is,

$$
\text { coannotation_score }_{-}=\sum \text { score }_{\text {leaf }}-\sum \text { score }_{\text {divergent_node }}
$$

If more than two leaves are derived from one divergent node, a weight score will be subtracted.
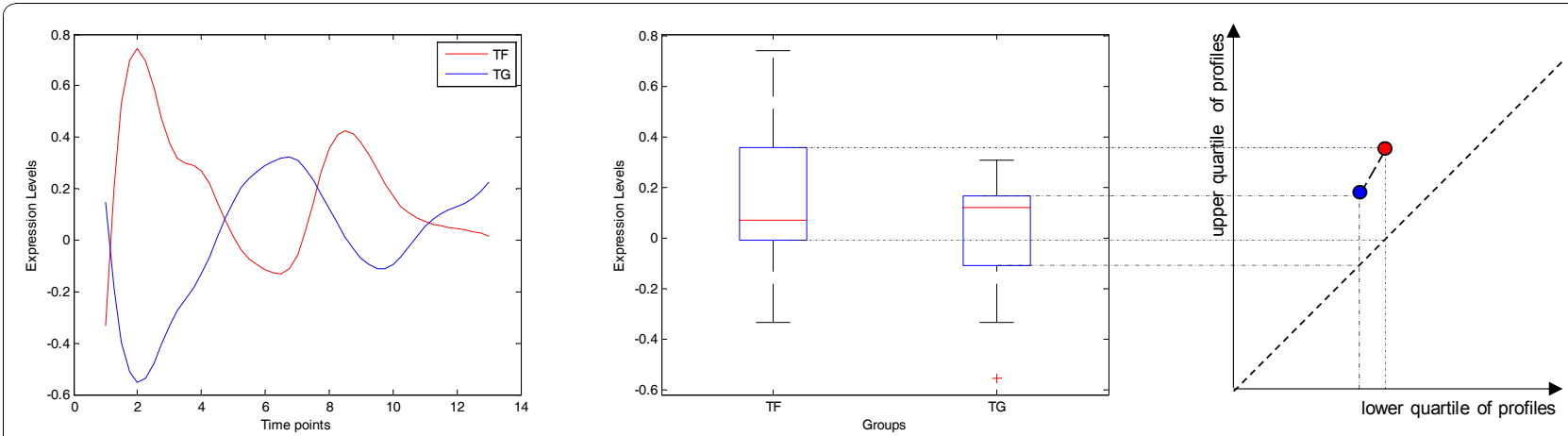

Figure 2 A mapping of the expression levels onto the variation space. The upper quartile of the expression levels of both TF and TG mapped onto the $y$-axis, as well as the lower quartile mapped onto the x-axis. And the Euclidean distance between the two mapping points was named the distance of expression levels' differences. 

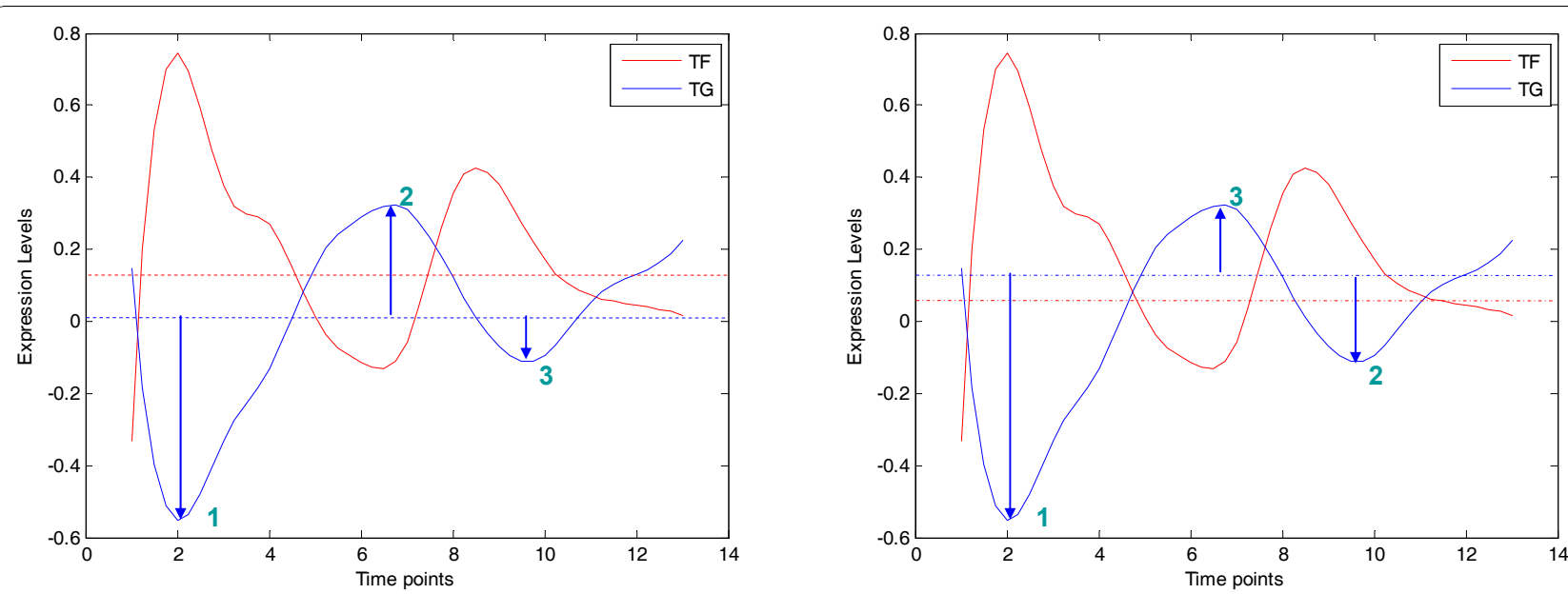

Figure 3 Illustration of vector analysis A) Mapping expression levels as sub-vectors (blue arrows) under each measurement in the coordination system of TF-TG's expression levels. B) Sum vector (red arrow) of sub-vectors. C) Quadrantal sum vector calculation. Measurements of expression relationships corresponding to the sub-vectors in different quadrants represent different patterns. Therefore, the sum vectors (orange arrows) were calculated in different quadrants respectively, and then we chose the vector with the largest modulus as the main representative vector (red arrow) of one TF-TG pair. E.L: expression levels

\section{Time-delay character for predicting of the direction of regulatory relationships}

During the transmission of expression perturbation from the TF to TG, a time delay might occur. Therefore, a suitable parameter that can describe the existence of the time delay might help to fix the direction of the regulatory relationship.

The point which has the extremum amplitude is a character that is to be considered. If the expression profile can be regarded as the continuous function of time, i.e., $y=f(t)$, the extremum exists at $y^{\prime}=\Delta y=\mathrm{d} y / \mathrm{d} t=0$. For discrete points such as the microarray time-series expression profile, this problem can be solved by difference, with the popularization of the differentiation. Boundary condition $\Delta y=0$ is often not available, so condition $\Delta y(t) \cdot \Delta y(t-1)<0$ might be more appropriate.

Besides, the basis of amplitude must be considered. Both the mean and median value might be available (Figure 4).

\section{Multiple features can be used to predict regulatory relations \\ Variation of expression levels}

By taking into account the ELD combined with widelyused PCC, the distribution of existing and non-existing regulatory relationship pairs can be classified more distinctly (Figure 5).

As shown in Figure 5, a significant classification could be found. The green points represent the non-existing pairs relatively concentrated in the region while the blue ones represent the existing regulatory pairs in this region. Judging by $\mathrm{PCC}$ alone without considering the parameter ELD would lead to more false negative judgments. Now the typical non-ideal cases described in the Additional file $1 \mathrm{C}$ and $\mathrm{D}$ might refuse to be classified falsely by PCC alone.

Furthermore, differences in standard deviation and mean value have a widely accepted definition and the accuracy of this parameter group is acceptable. So we also take the parameters $\Delta$ mean $-\Delta \delta$ as indicators. Generally, $\Delta$ mean $-\Delta \delta$ correspond to the likelihood ratio distribution represented by PCC. The accuracy of this parameter group is as high as that of ELD verified by J48 classification tree. However, it also has some cons, i.e. the parameters are pairwise, and these two parameters should be used at the same time.

\section{Modulus and angle of expression levels vector by vector analysis}

The vectors characterize the expression levels of one regulatory pair under each experiment condition. So it is common to calculate the sum vector of all the vectors. However, there are several different expression patterns such as correlation or inversion with or without time-shift. Sub-vectors of one regulatory gene pair in different quadrants should present different expression patterns. Therefore, sometimes sub-vectors in different quadrants may cancel out each other and result in a vain sum vector. Both the sum vector of random selected non-existing cases and that of existing cases might obtain counteractive results. After analysis of specific cases, we found that in different quadrants are distributed sub-vectors of vectors of an existing regulatory pair with perfectly synchronous expression profiles. And the sum vector of all the sub-vectors would be counteracted partly. See Figure 6a for detail. 


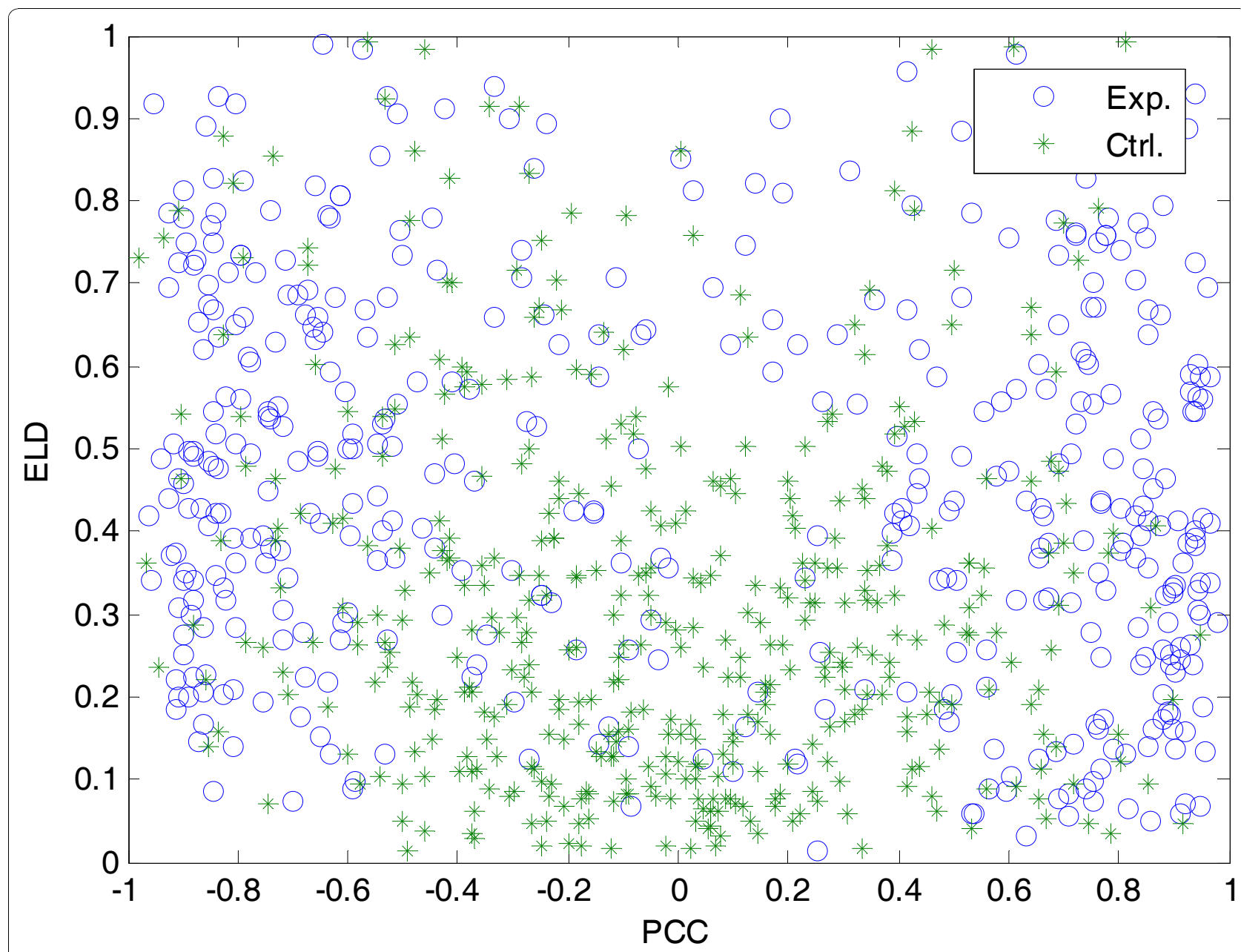

Figure 4 Distribution of different peaks based on mean and median values.

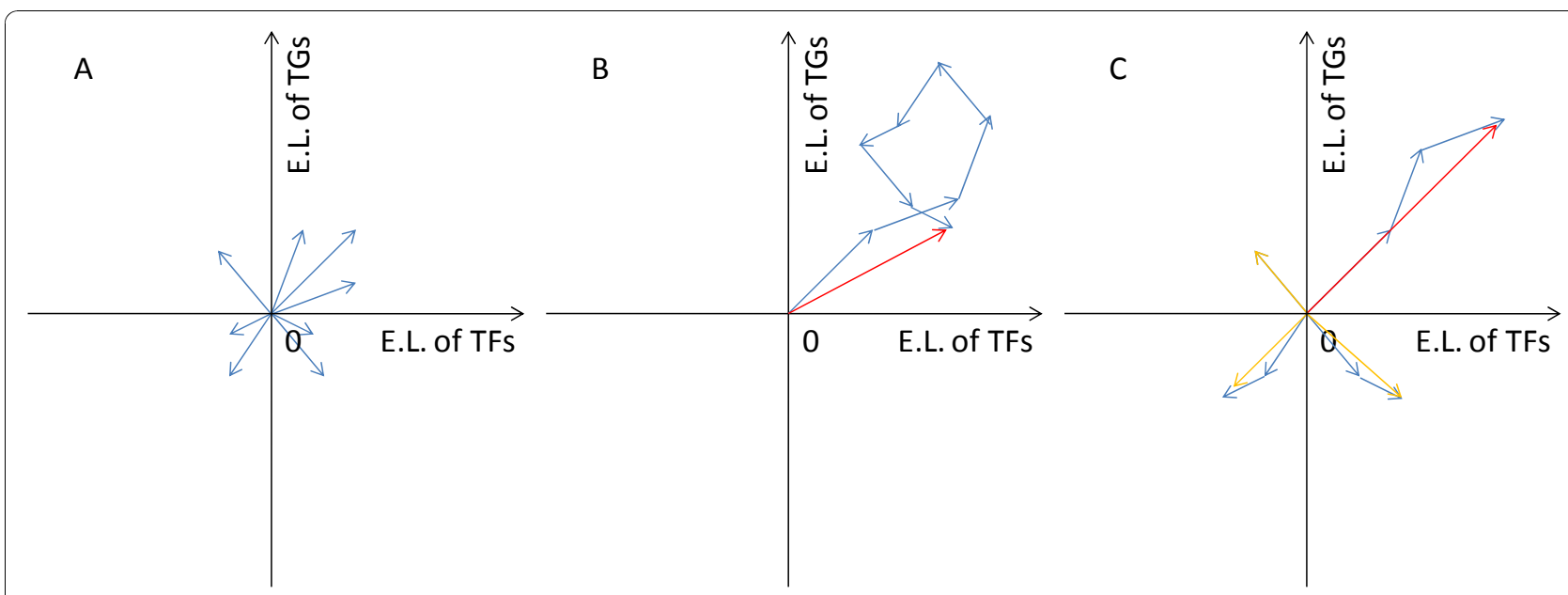

Figure 5 Distribution of regulation pairs of yeast cell cycle based on PCC and ELD. Non-existing pairs tend to cluster around the origin. Therefore, existing pairs with smaller $|P C C|$ but relatively larger ELD avoid classification into the non-existing group, which is quite natural if based on PCC alone. 


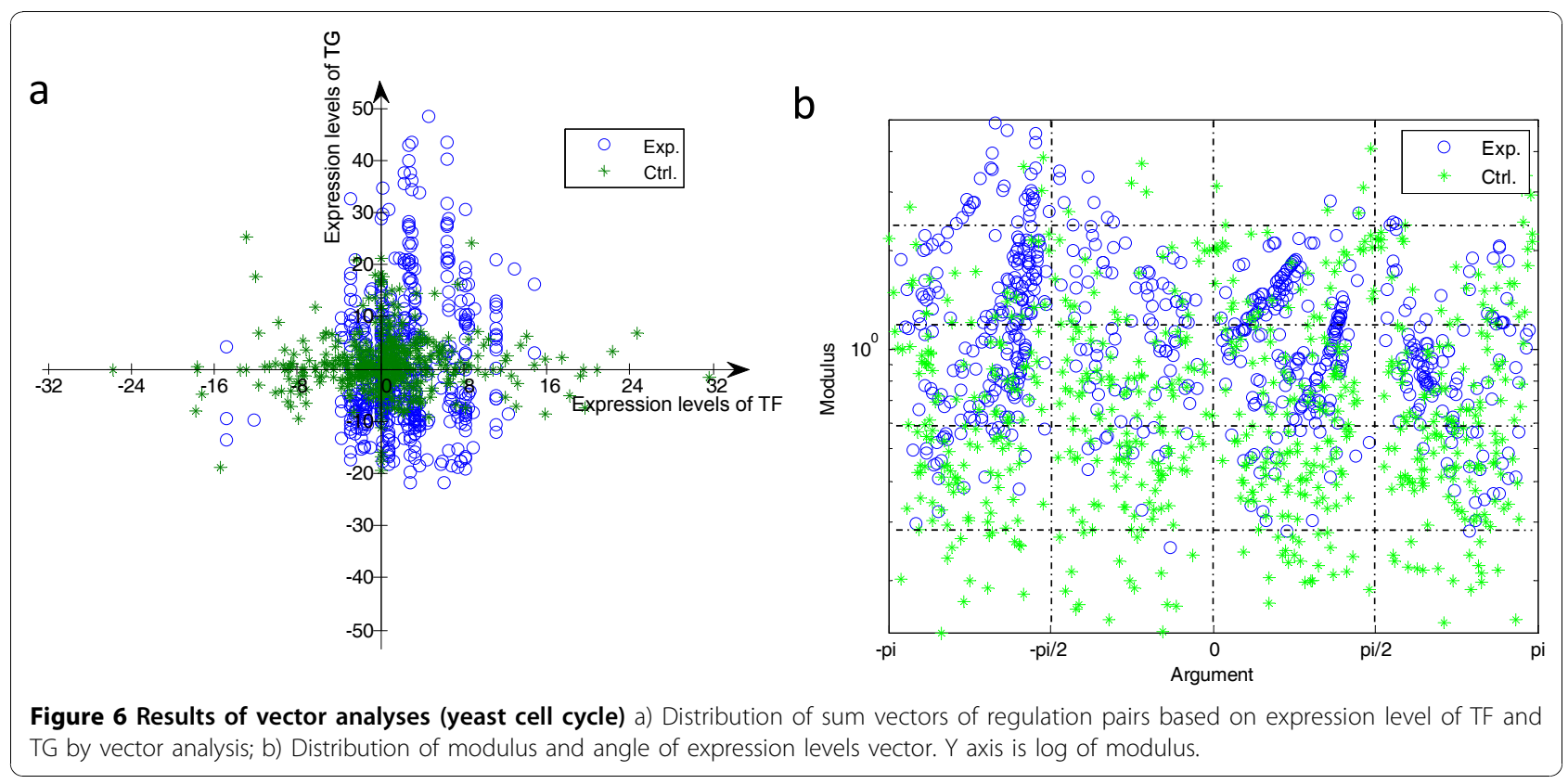

Given the properties of time-series gene expression relationships, it is natural to calculate the sum vectors in different quadrants respectively before choosing the vector which has the largest modulus as the main representative vector of the TF-TG pair. And the sum-vector should be mapped on the modulus-argument spaces (See Figure 6b).

Compared with non-quadrant vector analysis, quadrant vector analysis can yield a much more significant classification result. Additional file 2 shows the sum vectors of regulation pairs in the experiment group coloured by correlation of the expression level (PCC). In line with the meaning of $\mathrm{PCC}$, generally, main vectors in different quadrants indeed represent the different expression patterns of regulatory pairs.

\section{GO Coannotation}

We took GDS2318 dataset for example and calculated the GO scores. The results are shown in Figure 7.

As we expected, the frequency of the non-existing control cases declined while GO score increased. Meanwhile, the existing gene regulatory pairs showed increasing frequencies, suggesting that gene pairs with true regulatory relationship are more likely to emerge in the same biological process.

\section{Likelihood ratio}

The distribution of experimental and control groups according to the GO scores are perceptibly different, and it is easy to prove the existence of differences in distribution by statistical methods. However, the underlying meaning of the distribution has been left undisclosed. The positive likelihood ratio is a good option, for it could indicate the probability of the existence of the existing regulatory relationship and be used for integration of the parameters with Bayesian model.

First we calculated the likelihood ratio (LR), Figure 8.

As shown in Figure 8a, corresponding to the GO score, $L R$ s are higher in those bars where GO scores are higher, indicating that the probability is higher when GO score is relatively high as the positive likelihood ratio represents the probability. This result corresponds with the analysis above.

\section{The Bayesian model integrating multiple evidences is proved to be highly efficient Joint likelihood ratio}

Since some parameters we introduced are paired, the respective use of their $L R s$ might be unreasonable. Therefore we can combine the paired parameters to calculate the joint likelihood ratio of the joint parameter groups.

See Figure 9. Compared with Figure 5, the blue region near the origin shows low positive probability of the candidate pairs in this region. The regions with lower PCC and higher ELD are mainly coloured by red. This means that pairs located in these regions tend to be with true regulation. Given the characters of the expression levels, a possible explanation is that the existing pairs in these regions have different expression profiles. This character can be measured by the parameter ELD. The ELD of existing pairs with lower PCC is quite different from that of non-existing pairs. Compared with PCC or ELD individually, the joint likelihood ratio has a relatively strong discriminability. 

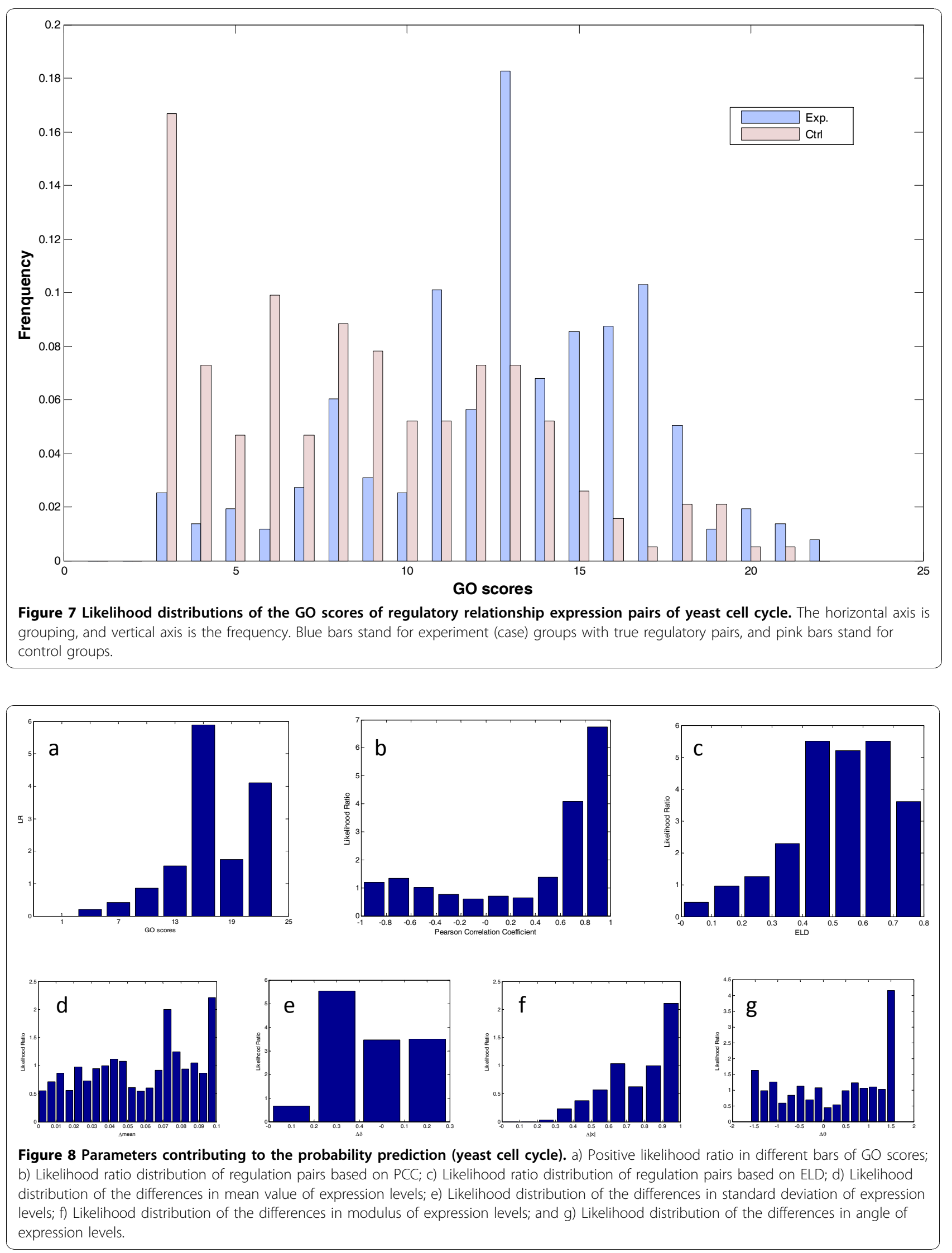


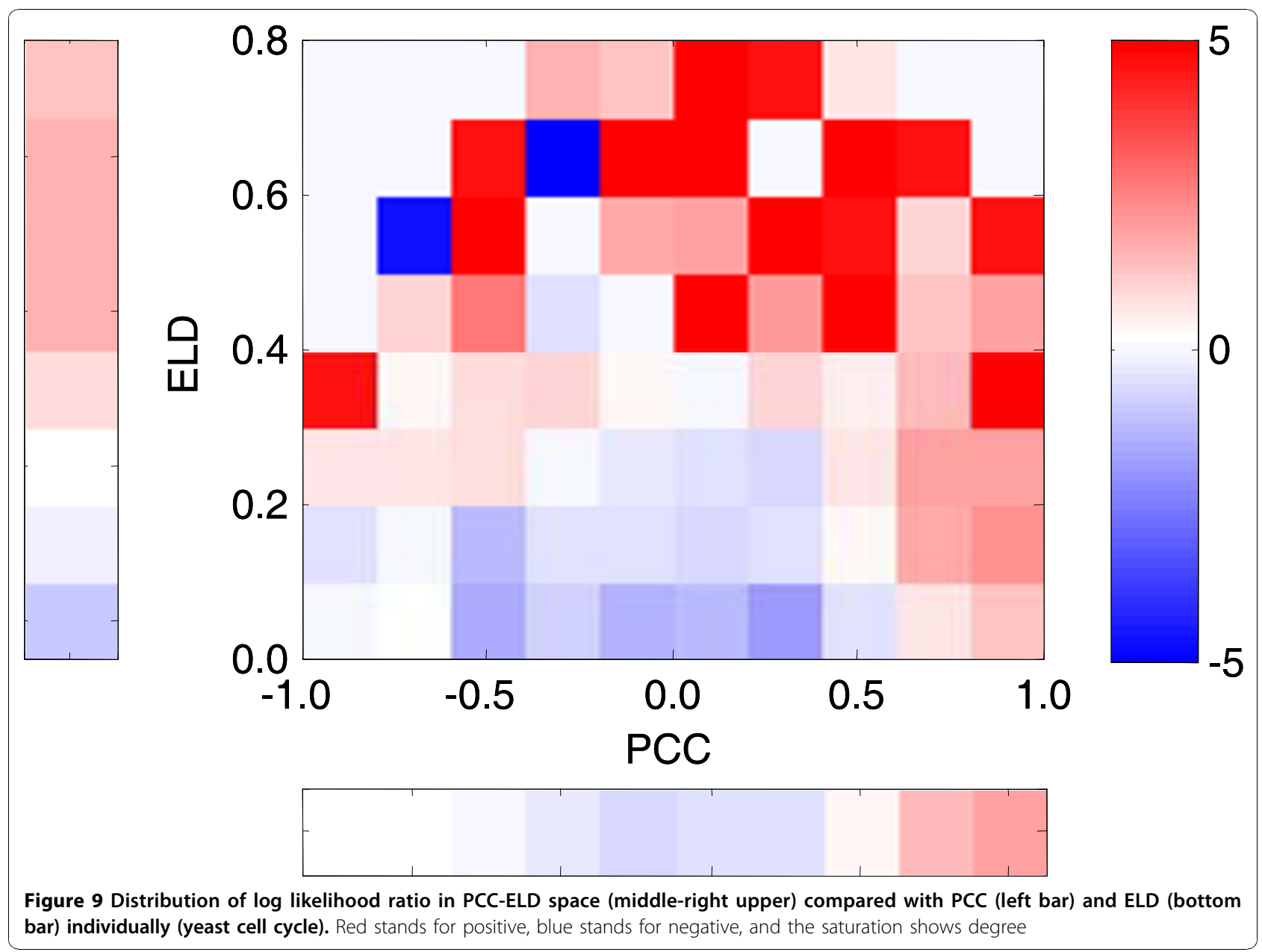

Besides, calculating joint likelihood ratio of ELD and PCC can save the trouble of proving the linear independence of PCC and ELD. And this method can be used for parameters groups e.g. modulus-angel groups naturally.

\section{Integration by Bayesian model}

In Figure 10, the resultant ROC curves [19] are illustrated. Each point on the ROC curve of each parameter denotes the sensitivity and specificity obtained from one test against a particular $L R_{\text {cutoff }}$. The area under the curve (AUC) of ROC is an indicator of the efficacy of the individual parameter or integrated model. An ideal test with $100 \%$ sensitivity and $100 \%$ specificity has an AUC 1.0, while a non-informative prediction has an area 0.5 , indicating it may be achieved randomly. The more the AUC of a test approximates 1.0, the higher the overall efficacy. We find that our improved Bayesian model has the largest AUC (0.8), which suggests it is better able to classify the true regulations against the test datasets. There is no doubt that the integrated model has the highest accuracy. For specific values of
AUC, please refer to Additional file 3. When the sensitivity is set at a relatively high value 0.8 , the specificity can reach up to 0.6 . When the specificity is set at a relatively high value 0.8 , the sensitivity can be 0.65 .

Time delay character for the prediction of the direction of regulatory relationships

Different numbers of peaks are tested respectively. Both mean and median values have been considered.

As showed in Figure 11, the condition with 2 peaks based on the amount of time-delay value is reasonable. The accuracy of the selected condition is 0.74 and the coverage is up to 0.93 .

\section{Comparison with other methods}

Typical existing methods include clustering algorithms, Bayesian networks, mutual information theory, as well as ordinary differential equations. Bansal et al. have compared these representative algorithms based on the simulated datasets [20].

We tested our approach on the same artificial datasets as [20], including both dynamic (time-series) and 


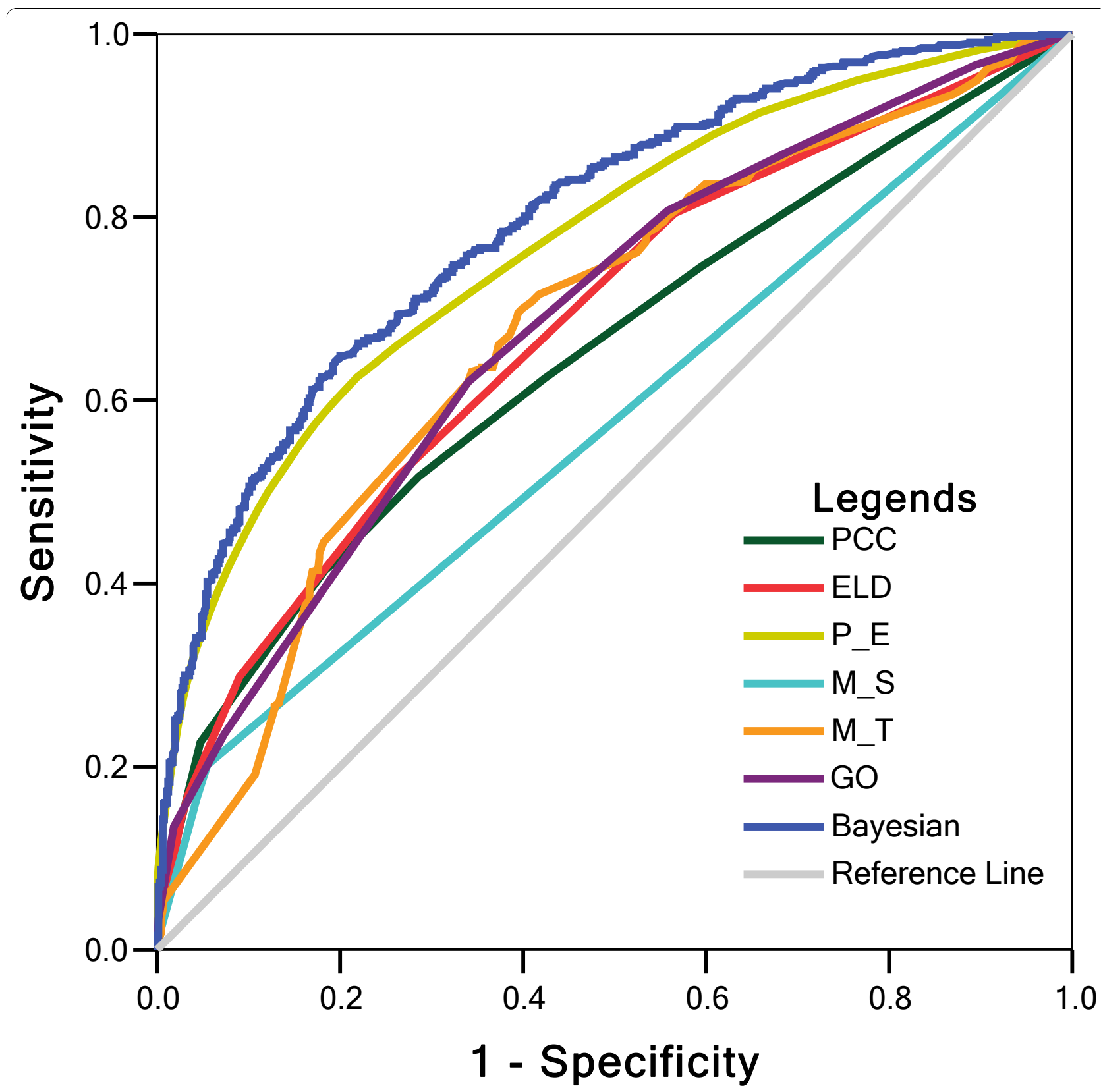

Figure 10 ROC curve for parameters and integrated Bayesian model using 5-fold cross-validations against the gold standard datasets (yeast cell cycle). P_E: PCC-ELD; M-S: $\triangle$ mean- $\Delta \delta, M_{-} T:|x|-\theta$.

steady-state (global and local perturbation) expression dataset. Genes in the artificial network are perturbed then measured at several time-series points to construct dynamic time series microarray data. And all the genes are perturbed and measured to generate global steadystate expression data. Local means only a single gene is perturbed during the artificial experiment. The results are shown in Table 1. According to adjusting threshold $L R$, we can get result of either PPV/Se priority, or keep balance of both of them.
Compared with algorithms tested by [20], our approach is better for dynamic (time-series) expression datasets. As shown in Table 1, in all these cases, while PPVs of our approach are equal to or slightly greater than those of the methods in [20], the corresponding Sensitivities are greater. For steady-state expression of global perturbation, our result is comparable with methods in [20]. Besides, our method performed on smaller network is somewhat better than that on the larger one. And the undirected predictions are slightly better than the directed ones. 


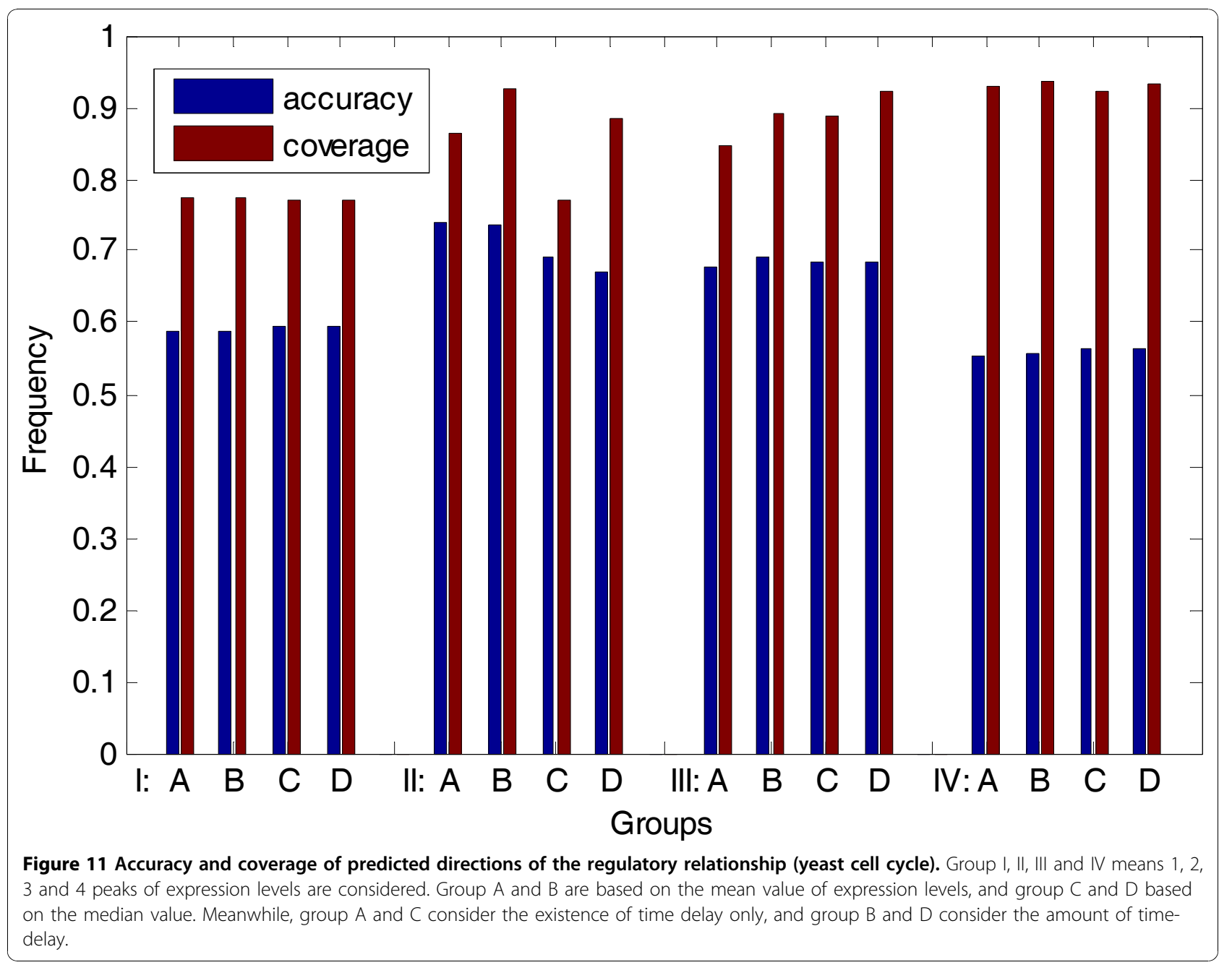

\section{Discussion}

In our research, besides the commonly used PCC, we proposed ELD, $\Delta$ mean $-\Delta \delta$, and $|x|-\theta$ as new parameters based on dynamic variation, as well as vector analysis. The parameter ELD represents the variation range character of the expression levels, and may prevent nonideal cases from false classification by PCC alone. Then, $\Delta$ mean- $\Delta \delta$ has an acceptable definition and accuracy. In vector analysis, we found that even the sub-vectors of a true regulatory gene pair with perfectly synchronous expression profiles are still distributed in different quadrants, and the sum vector might counteract partly. Vectors of one regulatory gene pair in different quadrants might represent different patterns. Therefore, we calculated the sum vectors in different quadrants respectively, and then chose the vector which has the largest modulus as the main representative vector of one TF-TG pair. Compared with non-quadrant vector analysis, the difference of distributions of modulus and argument is significant.
Also, we analyzed the functional co-annotation of transcription factors and their target genes, and then selected GO score as another parameter. As expected, the frequency of the non-existing control cases declined while GO score increased. Meanwhile, the existing gene regulatory pairs showed increasing frequencies, suggesting that gene pairs with true regulatory relationship have better chance of emerging in the same biological process.

Subsequently, we considered the Bayesian model for the likelihood ratio integration. Then the result was fairly acceptable. In our cases, some parameters we introduced are paired. We therefore combined the parameters to calculate the joint likelihood ratio of the joint parameter groups. The joint likelihood ratios of paired parameters make the $L R$ s seems reasonable and there is no need to prove the linear independence for the parameters.

Our approach is mainly based on several novel parameters, which could be intuitive indicators. We introduced 
Table 1 Result of our approach on simulated dataset

\begin{tabular}{|c|c|c|c|c|c|c|c|c|c|}
\hline \multirow[t]{2}{*}{ Conditions } & \multirow[t]{2}{*}{$\#$ of genes } & \multirow[t]{2}{*}{ \# of samples } & \multirow[t]{2}{*}{ Direction } & \multicolumn{2}{|c|}{ Se priority } & \multicolumn{2}{|c|}{ Balanced } & \multicolumn{2}{|c|}{ PPV priority } \\
\hline & & & & PPV & $\mathrm{Se}$ & PPV & $\mathrm{Se}$ & PPV & $\mathrm{Se}$ \\
\hline & 10 & 100 & u & 0.85 & 0.92 & 0.87 & 0.87 & 0.91 & 0.26 \\
\hline \multirow[t]{4}{*}{ Global } & & & $d$ & 0.41 & 0.89 & 0.51 & 0.78 & 0.71 & 0.63 \\
\hline & 100 & 1000 & u & 0.20 & 0.95 & 0.30 & 0.28 & 0.99 & 0.05 \\
\hline & & & $d$ & 0.11 & 0.93 & 0.25 & 0.16 & 0.93 & 0.09 \\
\hline & 10 & 10 & u & - & - & 0.58 & 0.85 & - & - \\
\hline \multirow[t]{4}{*}{ Local } & & & $d$ & 0.57 & 0.93 & 0.78 & 0.78 & 0.94 & 0.63 \\
\hline & 100 & 100 & u & 0.25 & 0.92 & 0.57 & 0.50 & 0.95 & 0.10 \\
\hline & & & $d$ & 0.18 & 0.89 & 0.33 & 0.60 & 0.97 & 0.10 \\
\hline & 10 & 100 & u & 0.47 & 0.97 & 0.67 & 0.79 & 0.93 & 0.63 \\
\hline \multirow[t]{3}{*}{ Dynamic } & & & $d$ & 0.49 & 0.96 & 0.64 & 0.67 & 0.92 & 0.48 \\
\hline & 100 & 1000 & u & 0.21 & 0.90 & 0.42 & 0.23 & 0.55 & 0.10 \\
\hline & & & $d$ & 0.11 & 0.91 & 0.39 & 0.21 & 0.94 & 0.11 \\
\hline
\end{tabular}

Both dynamic and steady-state (Global and local) expression dataset has been tested. Please refer to Section "Comparison with other methods" for the "conditions" description of "Global", "Local" and "Dynamic". PPV: positive predicted value; Se: sensitivity; u: undirected; d: directed.

these parameters to describe characters of microarray expression data of regulating gene pairs. These features include the variation of expression level, the divergence of statistical characters, and the consistent degree of representative measurements. Additionally, our approach is much less costly than some mainstream methods. Therefore, our approach can serve as a fast pre-process strategy for microarray data analysis.

Some papers argue that inferring regulatory relationship based on microarray has inherent faults. First, the similarity of the expression profile suggests nothing more than a statistical dependency between two genes, not a direct causal relationship. The verification of the relationship requires other evidences, such as ChIP-chip data, $\mathrm{Y} 2 \mathrm{H}$ or other wet experiments. Second, essential genes [21] which are always expressed in the cell cannot be disturbed by knockdown or knockout. Therefore microarray experiments do not work well on these essential genes. Third, microarray is a kind of highthroughput analysis technology after all, so it cannot be very precise. Genes with a slim expression level can hardly be detected accurately.

Recently a series of reports indicates that the microarray might be replaced by fast high-throughput sequencing $[22,23]$, which, however, cannot be made as inexpensive and efficient as microarray now. In the future, microarray might be used to meet more specific research needs, such as fast elementary filter or test. Therefore complex models might not be suited to the fast measurement of the microarray. Though our approach is more or less rough and far from perfect, we still believe some simple indicators based on uncomplicated characters would reveal complex behaviour.

\section{Conclusions}

With the rapid deposition of the microarray data in recent years, microarray data have become an increasingly important data source for bioinformatics research. On the basis of microarray data, constructing gene regulatory networks has also become a hotspot. By constructing the gene regulatory network, we can identify the complicated regulatory relationships, uncover the regulatory patterns in the cell, and gain the global view of the biological process. In this paper, we present some novel parameters to uncover potential characters of regulatory relationships. In addition to routine description of the similarity of the expression levels, our proposed parameters measured range of the variation and the statistic feature of expression levels, consistency of sub-vectors of the expression level, as well as functional co-annotation of regulating pairs. Unlike other global expression profiles computational methods, our approach is mainly based on several novel parameters, which could be intuitive indicators. And our parametric approach can serve as a fast approach for regulatory relationship mining.

\section{Materials and methods Datasets}

As a simple but important organism, yeast Saccharomyces cerevisiae is a proper target of research. First we set up an experiment group of regulatory element pairs with existing (true) regulatory relationship. These existing pairs were obtained from published literature [24,25]. In addition, we constructed a control group for training dataset. The pairs in control group were randomly selected and known existing regulatory pairs had been excluded. During the research, we observed that the ratio of existing and 
non-existing pairs in the training set would affect the result. The increases of negative data in training set induce a decrease of positive prediction value with the fixed sensitivity. It indicates that suitable ratio of positive and negative must be noticed. The result is shown in Additional file 4. Time series datasets derived from cell cycle experiments were downloaded from GEO dataset in PubMed. The GO annotations are retrieved by GOfact [26]. For a proper comparison with other methods, artificial datasets is an appropriate choice. In silico data could control the noise levels of the data. Here, we used the datasets in [20], which was generated an artificial dataset by linear ODEs, with the mean of white noise 0 and standard deviation 0.1 .

\section{Bayesian model}

Bayesian model has been widely used for integrating proofs $[27,28]$. Likelihood ratio is the probability of observing an existing gene pair in predictive datasets divided by the probability of observing the non-existing gene pair in predictive datasets [29]. Here prior odds are the chance of choosing a pair of regulatory genes from all candidate gene pairs.

Posterior odds of regulation is the product of prior odds and likelihood ratio.

$$
O_{\text {post }}=\frac{P(\text { positive } \mid f)}{P(\text { negative } \mid f)}=O_{\text {prior }} \times L R(f)
$$

The prior odds of regulation are the probability of occurrence of the positive divided by the probability of occurrence of the negative.

$$
O_{\text {prior }}=\frac{P(\text { positive })}{P(\text { negative })}
$$

In other words, $P$ (positive) is the probability of finding a pair of genes in all the possible regulation, and the $P$ (negative) is the probability of finding a non-regulation pair in all the possible regulation. The posterior odds are often decided by the mean numbers of regulation in all the known regulation. So the posterior odds are

$$
O_{\text {post }}=\frac{P\left(\text { positive } \mid f_{1} \ldots f_{n}\right)}{P\left(\text { negative } \mid f_{1} \ldots f_{n}\right)}
$$

$f_{\mathrm{i}}$ means the number of gene pairs in the dataset $\mathrm{i}$. And the Bayesian method is considered

$O_{\text {post }}=O_{\text {prior }} \times L R\left(f_{1} \ldots f_{n}\right)$

Subsequently,

$$
L R=\frac{P\left(f_{1} \ldots f_{n} \mid \text { positive }\right)}{P\left(f_{1} \ldots f_{n} \mid \text { negative }\right)}
$$

In this formula, $L R$ means likelihood ratio, and positive means gold standard positive dataset of gene pairs where real regulatory relationship exists. And negative is the gold standard negative dataset in which no gene pair has any regulation.

Finally, under certain assumptions, such as the predicted dataset, individual or non-redundant, the likelihood can be counted by the product of likelihoods of individual sets.

$$
L R=\prod_{i=1}^{i=n} \frac{P\left(f_{i} \mid \text { positive }\right)}{P\left(f_{i} \mid \text { negative }\right)}
$$

This is also known as the naïve Bayesian network.

Additional file 1: Left: experience group. A) An ideal expression profile of coregulatory pair with a relatively higher $\mid$ PCC. C) An expression profile of a true regulatory pair with a lower |PCC|. Right: control group. B) A typical expression profile of a non-existing pair with a lower |PCC|. D) A non-existing pair whose variation ranges of expression levels are relatively smaller, reduced a relatively higher $|\mathrm{PCC}|$.

Additional file 2: E.L.: expression level. Red stands for positive correlation, blue stands for negative correlation, and the saturation shows the correlation degree. Sum vectors of regulation pairs in experiment group were coloured by PCC. Compared with the meaning of PCC, generally, main vectors in different quadrants indeed represent the different expression patterns of regulatory pairs.

Additional file 3: Our proposed model has a relative large AUC (0.8), which suggests it is able to predict regulations efficiently.

Additional file 4: PPV1 to PPV7 in scales stand for positive and negative ratio in training set is 1:1 to $1: 7$; and the scale "PPV" stands for all the pairwised cases in the network composed by all the genes have been considered.

\section{List of abbreviations}

AUC: area under the curve; ELD: expression level differences; GEO: Gene Expression Omnibus; GO: Gene Ontology; LR: likelihood ratio; ODE: ordinary differential equation; PCC: Pearson correlation coefficient; PPV: positive predictive value; ROC: receiver operating characteristic; Se: sensitivity; TF: transcriptional factor; TG: target gene

\section{Acknowledgements}

This work was supported by the Chinese National Key Program of Basic Research [grant numbers 2006CB910803, 2010CB912700 and 2011CB910202], the National High Technology Research and Development Program of China [grant number 2006AA02A312], National Science and Technology Major Project [grant numbers 2008ZX10002-016, 2009ZX09301-002], National Natural Science Foundation of China [grant numbers 30621063, 30800200]. This article has been published as part of BMC Bioinformatics Volume 11 Supplement 11, 2010: Proceedings of the 21st International Conference on Genome Informatics (GIW2010). The full contents of the supplement are available online at http://www.biomedcentral.com/1471-2105/11?issue=S11.

\section{Author details}

${ }^{1}$ State Key Laboratory of Proteomics, Beijing Proteome Research Center, Beijing Institute of Radiation Medicine, Beijing 102206, China. ²Department of Chemistry and Biology, College of Science, National University of Defense Technology, Changsha 410073, China.

\section{Authors' contributions}

W. Liu and D. Li designed and implemented the whole methodology and the computation framework. Q. Liu provided constructive discussions and 
refinement of the formula. Y. Zhu monitored the whole framework and revised the manuscripts. F. He directed the whole project and revised the manuscript. All the authors have read and agreed to the manuscript.

\section{Competing interests}

The authors declare that they have no competing interests.

Published: 14 December 2010

\section{References}

1. Yu H, Luscombe NM, Qian J, Gerstein M: Genomic analysis of gene expression relationships in transcriptional regulatory networks. Trends Genet 2003, 19(8):422-427.

2. Eisen MB, Spellman PT, Brown PO, Botstein D: Cluster analysis and display of genome-wide expression patterns. Proc Natl Acad Sci U S A 1998, 95(25):14863-14868.

3. Amato R, Ciaramella A, Deniskina N, Del Mondo C, di Bernardo D, Donalek C, Longo G, Mangano G, Miele G, Raiconi G, et al: A multi-step approach to time series analysis and gene expression clustering. Bioinformatics 2006, 22(5):589-596.

4. Toh $\mathrm{H}$, Horimoto $\mathrm{K}$ : Inference of a genetic network by a combined approach of cluster analysis and graphical Gaussian modeling. Bioinformatics 2002, 18(2):287-297.

5. Yan X, Mehan MR, Huang Y, Waterman MS, Yu PS, Zhou XJ: A graph-based approach to systematically reconstruct human transcriptional regulatory modules. Bioinformatics 2007, 23(13):i577-586.

6. Huang S: Gene expression profiling, genetic networks, and cellular states: an integrating concept for tumorigenesis and drug discovery. J Mol Med 1999, 77(6):469-480.

7. Martin S, Zhang Z, Martino A, Faulon JL: Boolean dynamics of genetic regulatory networks inferred from microarray time series data. Bioinformatics 2007, 23(7):866-874.

8. Kim H, Lee JK, Park T: Boolean networks using the chi-square test for inferring large-scale gene regulatory networks. BMC Bioinformatics 2007, 8:37.

9. Friedman N, Linial M, Nachman I, Pe'er D: Using Bayesian networks to analyze expression data. J Comput Biol 2000, 7(3-4):601-620.

10. Pe'er D, Regev A, Elidan G, Friedman N: Inferring subnetworks from perturbed expression profiles. Bioinformatics 2001, 17(Suppl 1):S215-224.

11. Husmeier D: Sensitivity and specificity of inferring genetic regulatory interactions from microarray experiments with dynamic Bayesian networks. Bioinformatics 2003, 19(17):2271-2282.

12. Zou M, Conzen SD: A new dynamic Bayesian network (DBN) approach for identifying gene regulatory networks from time course microarray data. Bioinformatics 2005, 21(1):71-79.

13. Bansal M, Gatta GD, di Bernardo D: Inference of gene regulatory networks and compound mode of action from time course gene expression profiles. Bioinformatics 2006, 22(7):815-822.

14. $\mathrm{Vu} T \mathrm{~T}$, Vohradsky J: Nonlinear differential equation model for quantification of transcriptional regulation applied to microarray data of Saccharomyces cerevisiae. Nucleic Acids Res 2007, 35(1):279-287.

15. Margolin AA, Nemenman I, Basso K, Wiggins C, Stolovitzky G, Dalla Favera R, Califano A: ARACNE: an algorithm for the reconstruction of gene regulatory networks in a mammalian cellular context. $B M C$ Bioinformatics 2006, 7(Suppl 1):S7.

16. Butte AJ, Kohane IS: Mutual information relevance networks: functional genomic clustering using pairwise entropy measurements. Pac Symp Biocomput 2000, 418-429.

17. Steuer R, Kurths J, Daub CO, Weise J, Selbig J: The mutual information: detecting and evaluating dependencies between variables. Bioinformatics 2002, 18(Suppl 2):S231-240.

18. Breitling $R$, Armengaud $P$, Amtmann A: Vector analysis as a fast and easy method to compare gene expression responses between different experimental backgrounds. BMC Bioinformatics 2005, 6:181.

19. Baldi P, Brunak S, Chauvin Y, Andersen CA, Nielsen H: Assessing the accuracy of prediction algorithms for classification: an overview. Bioinformatics 2000, 16(5):412-424

20. Bansal M, Belcastro $V$, Ambesi-Impiombato A, di Bernardo D: How to infer gene networks from expression profiles. Mol Syst Biol 2007, 3:78
21. Mnaimneh S, Davierwala AP, Haynes J, Moffat J, Peng WT, Zhang W, Yang X, Pootoolal J, Chua G, Lopez A, et al: Exploration of essential gene functions via titratable promoter alleles. Cell 2004, 118(1):31-44.

22. Ledford H: The death of microarrays? Nature 2008, 455(7215):847.

23. Shendure J: The beginning of the end for microarrays? Nat Methods 2008 , 5(7):585-587.

24. Lee TI, Rinaldi NJ, Robert F, Odom DT, Bar-Joseph Z, Gerber GK, Hannett NM, Harbison CT, Thompson CM, Simon I, et al: Transcriptional regulatory networks in Saccharomyces cerevisiae. Science 2002, 298(5594):799-804

25. Luscombe NM, Babu MM, Yu H, Snyder M, Teichmann SA, Gerstein M: Genomic analysis of regulatory network dynamics reveals large topological changes. Nature 2004, 431(7006):308-312.

26. Li D, Li J, Ouyang S, Wang J, Xu X, Zhu Y, He F: An Integrated Strategy for Functional Analysis in Large-scale Proteomic Research by Gene Ontology. Progress in Biochemistry and Biophysics 2005, 32(11):1026-1029.

27. Jansen R, Yu H, Greenbaum D, Kluger Y, Krogan NJ, Chung S, Emili A, Snyder M, Greenblatt JF, Gerstein M: A Bayesian networks approach for predicting protein-protein interactions from genomic data. Science 2003, 302(5644):449-453.

28. Rhodes DR, Tomlins SA, Varambally S, Mahavisno V, Barrette T, KalyanaSundaram S, Ghosh D, Pandey A, Chinnaiyan AM: Probabilistic model of the human protein-protein interaction network. Nat Biotechnol 2005, 23(8):951-959.

29. Eddy SR: What is Bayesian statistics? Nat Biotechnol 2004, 22(9):1177-1178.

doi:10.1186/1471-2105-11-S11-S15

Cite this article as: Liu et al: A novel parametric approach to mine gene regulatory relationship from microarray datasets. $B M C$ Bioinformatics 2010 11(Suppl 11):S15.

\section{Submit your next manuscript to BioMed Central and take full advantage of:}

- Convenient online submission

- Thorough peer review

- No space constraints or color figure charges

- Immediate publication on acceptance

- Inclusion in PubMed, CAS, Scopus and Google Scholar

- Research which is freely available for redistribution

Submit your manuscript at www.biomedcentral.com/submit
Ciomed Central 